\title{
Evaluación de espinillo como abono verde en la producción de biomasa y proteína de acelga
}

\author{
Evaluation of gorse as green manure in the production \\ of biomass and protein chard
}

\author{
Ricardo Tighe $^{1 *}$, Gina Leonelli ${ }^{1}$, Macarena Aliaga ${ }^{1}$, Mario Rodríguez $^{1}$
}

\section{RESUMEN}

Se evaluó Ulex europaeus como abono verde sobre la producción de biomasa y proteína en acelga (Beta vulgaris L. var. Cicla). Los tratamientos fueron 1,6 kg de Lupinus albus; $1,6 \mathrm{~kg}$ de $U$. europaeus y un testigo absoluto. Las unidades experimentales fueron parcelas de $2 \mathrm{~m}^{2}$, dispuestas en bloques completamente al azar, con cuatro repeticiones. Los datos se sometieron a un ANDEVA y a la prueba de Tukey con 5\% de significancia. Transcurridos 60 días de la incorporación, se establecieron plántulas de B. vulgaris var. Cicla. La producción de biomasa y análisis proximal se evaluó a los 120 días del establecimiento; y el análisis de suelo, a los 184 días de la incorporación. U. europaeus generó un $42 \%$ más de materia verde respecto del testigo ( $\leq \leq 0,05)$, sin diferencias con lupino ( $>0,05)$. No se encontraron diferencias $(\mathrm{p}>0,05)$ en la producción de materia seca. El contenido de proteína fue $14 \%$ superior $(\mathrm{p} \leq 0,05)$ en $U$. europaeus y L. albus respecto del testigo. El nivel de NPK en el suelo fue mayor en U. europaeus. El aumento de materia verde y proteína en $B$. vulgaris var. Cicla, así como de NPK en el suelo, avalan el uso de U. europaeus como abono verde.

Palabras clave: Beta vulgaris var. Cicla, materia verde, Lupinus albus, Ulex europaeus.

\begin{abstract}
We assessed the response of biomass and protein production of chard (Beta vulgaris L. var. Cicla.), by using Ulex europaeus vs. Lupinus albus as green manure. Treatments were $1.6 \mathrm{~kg}$ of $\mathrm{L}$. albus; $1.6 \mathrm{~kg}$ of $\mathrm{U}$. europaeus and absolute control. The experimental units were $2 \mathrm{~m}^{2}$ plots arranged in a randomized complete block with four replications. The data were analyzed by ANOVA and Tukey's tests at 5\% significance. Plants of chard were established after 60 days of the incorporation of the green manure. Biomass production as well as proximal analysis was performed at 120 days after establishment. In addition, a soil analysis was performed at 184 after incorporation of green manure. The utilization of $\mathrm{U}$. europaeus improved in $42 \%$ fresh biomass production when compared with the control $(p \leq 0.05)$, however no differences were observed when it was compared with lupin $(p>0.05)$. No differences were observed for dry matter production among the treatments $(p>0.05)$. Water content it was $14 \%$ greater for treatments that included green manure. The levels of NPK in the soil were greater for U. europaeus treatment. The increase of green matter and protein in chard as well as the increase of NPK levels in the soil, support the use of U. europaeus as green manure.
\end{abstract}

Key words: Beta vulgaris var. Cicla, fresh biomass, Lupinus albus, Ulex europaeus.

\section{Introducción}

El abono verde es la incorporación de vegetales, generalmente leguminosas, en estado verde al suelo sin previa descomposición. El propósito es mejorar las condiciones físicas, químicas y biológicas del sustrato, siendo una fuente importante de $\mathrm{N}$ y $\mathrm{C}$ en los sistemas de cultivos (Chi-Peng et al., 2014). Contribuye a incrementar la asimilabilidad del fósforo y oligoelementos, por lo que su descomposición mejora las condiciones edáficas (Eriksen, 2005).
También tiene utilidad como cobertura, protegiendo el suelo de la erosión, la compactación por la lluvia y la pérdida de humedad por evapotranspiración, contribuyendo a su restauración (Tejada et al., 2008). Ha sido demostrada la potencialidad de los abonos verdes para sustituir parcial o totalmente las necesidades de nitrógeno e incrementar los rendimientos de numerosos cultivos (Eriksen, 2005; Tejada et al., 2008).

El uso de leguminosas es recomendable, ya que tienen la capacidad de fijar nitrógeno, lo que

\footnotetext{
1 Universidad Católica de Temuco, Facultad de Recursos Naturales, Escuela de Agronomía. Rudecindo Ortega 02950, Temuco, Chile.

* Autor para correspondencia: rtighe@uct.cl
}

Fecha de Recepción: 20 Octubre, 2014.

Fecha de Aceptación: 24 Marzo, 2015. 
incrementa su contenido y posterior aporte al suelo (Crews y Peoples, 2004).

El lupino (Lupinus albus L.) es una leguminosa que tradicionalmente se ha cultivado, entre otras razones, para abono verde (Rosolem et al., 2002; Jensen et al., 2004), generando aportes nutricionales de 13,$6 ; 1,4 ; 10,8 \mathrm{mg} \mathrm{kg}^{-1}$ de NPK, respectivamente (García et al., 2002). Debido a su carácter productivo existe la necesidad de otras especies con propiedades similares que puedan ser utilizadas para elaborar abono verde. En este marco, la leguminosa espinillo (Ulex europaeus L.) reúne características que hacen plausible su consideración. U. europaeus es una de las malezas más invasoras que impide el desarrollo de otras especies (Quiroz et al., 2009). Es considerada una maleza agresiva en Nueva Zelanda, los EE.UU., Chile y Australia (Norambuena et al., 2007; Hill et al., 2008). Puede desarrollarse bien en terrenos deficientes de calcio, tiende a acidificar el sustrato y bajar la capacidad de intercambio catiónico de los suelos moderadamente fértiles (MacCarter y Gaynor, 1980). Es una especie de amplia distribución, se ha registrado su presencia en más de 50 países e islas (Hill et al., 2008). En Chile está presente desde la Región de Valparaíso hasta la de Los Lagos, invadiendo suelos arenosos y arcillosos, pobres y degradados (Quiroz et al., 2009).

$U$. europaeus posee una alta capacidad de producción alcanzando $6.000 \mathrm{~kg} \mathrm{MS} \mathrm{ha}^{-1}$ en plantas de un año de edad y $77.000 \mathrm{~kg} \mathrm{MS} \mathrm{ha}^{-1}$ al décimo año (Meeklah, 1979). Según Howe et al. (1988), su composición nutricional es: $\mathrm{N} \mathrm{4,8 \%} \mathrm{a} \mathrm{3,1 \% ;} \mathrm{P}$ $0,32 \%$ a $0,48 \%$; K $3,1 \%$ a $1,8 \%$; S $0,24 \%$ a $0,18 \%$; Ca $0,69 \%$ a $0,51 \% ; \mathrm{Mg} 0,48 \%$ a $0,3 \% ; \mathrm{Na} 0,5 \%$ a $0,24 \%$ y para micronutrientes se han reportado valores de $\mathrm{Cu} 6 \%$ a $8 \%$; $\mathrm{Fe} 62 \%$ a $59 \%$; Mn $84 \%$ a $71 \%$; Zn $36 \%$ a $44 \%$. Presenta una relación C:N moderada (López y López, 2012). Dada su capacidad fijadora de nitrógeno y a su eficiencia fotosintética, permite acumular abundante biomasa rica en $\mathrm{N}$, componente que solo se torna útil para otras especies cuando $U$. europaeus se descompone naturalmente. La forma tradicional de eliminar a $U$. europaeus es la quema, liberándose grandes cantidades de calcio, magnesio, potasio y fósforo, mientras que la mayoría del nitrógeno se pierde (Mac Carter y Gaynor, 1980).

El abono verde se utiliza en cultivos que requieren de alta fertilidad y contenido de materia orgánica. Un grupo de ellos son los hortícolas, donde destaca la acelga (Beta vulgaris var. Cicla).
Al respecto, Dzida et al. (2012) afirman que el principal factor que afecta al tamaño, calidad y rendimiento es la fertilización nitrogenada.

De acuerdo con los antecedentes expuestos, $U$. europaeus podría constituir una alternativa para la elaboración de abono verde con resultados similares a los presentados por L. albus. Debido a lo anterior, se plantea evaluar el efecto del abono verde de $U$. europaeus en la producción de biomasa y contenido de proteína en acelga, y características químicas del suelo.

\section{Materiales y Métodos}

El ensayo se realizó entre enero y agosto de 2012 en dependencias de la empresa Agroindustrias Daube Ltda., ubicada en camino Botrolhue km 5,5 Temuco, Chile. Los análisis se realizaron en el Laboratorio de Bromatología de la Universidad Católica de Temuco, Escuela de Agronomía; y en el Laboratorio de Suelos Labosel UCT Ltda., Temuco, Chile.

Para la elaboración de abono verde se utilizó lupino (L. albus) y Ulex (U. europaeus). El primero fue recolectado en el sector Rulo, comuna de Nueva Imperial, Chile; y el segundo, en el Centro Experimental Pillanlelbún de la Universidad Católica de Temuco, comuna de Lautaro, Chile.

La materia verde incorporada se obtuvo y cuantificó desde un cultivo de L. albus en floración destinado para abono verde. Basándose en ello se estableció una relación biomasa / superficie, equivalente a $0,8 \mathrm{~kg} \mathrm{~m}^{-2}$ que fue considerada en cada tratamiento (Tabla 1). La materia verde de U. europaeus se obtuvo de brotes nuevos en plantas adultas. Todo el material vegetal fue triturado manualmente, con tijera de podar, a un tamaño promedio de $3 \mathrm{~cm}$. Posteriormente, el material fue incorporado en parcelas de $2 \mathrm{~m}^{2}$ a $20 \mathrm{~cm}$ de profundidad.

El trasplante de acelga (B. vulgaris var. Cicla) se realizó 97 días después de incorporado el abono verde. Se consideraron plántulas de $10 \mathrm{~cm}$ de altura.

Tabla 1. Tratamientos del experimento.

\begin{tabular}{clc}
\hline Tratamiento & \multicolumn{1}{c}{ Descripción } & $\begin{array}{c}\text { Biomasa } \\
\text { incorporada } \\
\left(\mathrm{kg} \mathrm{2} \mathrm{m}^{-2}\right)\end{array}$ \\
\hline T0 & Testigo & 0 \\
T1 & Abono verde de U. europaeus & 1,6 \\
T2 & Abono verde de L. albus & 1,6 \\
\hline
\end{tabular}


Se efectuó un análisis de suelo a cada tratamiento el día 184 después de incorporado el material vegetal y 87 días después de establecido el cultivo. Las muestras se obtuvieron de la homogeneización de 10 submuestras, las que fueron sometidas a un análisis de rutina en el Laboratorio de Suelos Labosel.

Transcurridos 120 días del trasplante de $B$. vulgaris var. Cicla, se cosecharon cuatro plantas centrales de cada parcela, las que fueron rotuladas y llevadas al Laboratorio de Bromatología de la Universidad Católica de Temuco.

Las variables de respuesta fueron: materia seca de acelga (MS), materia verde de acelga (MV), análisis proximal y determinación de proteína cruda (PC) de acelga. La MS se determinó por deshidratación de la MV en estufa de aire forzado (Memmert 800) a $60{ }^{\circ} \mathrm{C}$ por $24 \mathrm{~h}$. El \% MS se calculó de la relación entre MS y MV.

La PC se determinó siguiendo la metodología recomendada por la AOAC (2011) y el extracto etéreo (EE) se obtuvo por el método Goldfish (AOAC, 2011) mediante un extractor (Labconco, 600lf).

El contenido de fibra cruda (FC) se determinó por gravimetría mediante la incineración del residuo de la digestión con soluciones de ácido sulfúrico e hidróxido de sodio. Finalmente, la determinación de cenizas se realizó mediante la calcinación a $550{ }^{\circ} \mathrm{C}$ en un horno mufla (Termolyne, 62700 Furnace).

El diseño experimental fue de bloques completamente al azar con tres tratamientos y cuatro repeticiones. Los datos fueron sometidos a un análisis de varianza (ANDEVA) y posteriormente a la prueba de comparación múltiple de Tukey con un 5\% de significancia. Los datos expresados en porcentajes fueron transformados mediante la raíz cuadrada del porcentaje. Se utilizó el programa estadístico JMP 5.0.1. (SAS, 2003).

\section{Resultados y Discusión}

En relación con la producción de biomasa de $B$. vulgaris var. Cicla, la MV fue $42 \%$ mayor $(\mathrm{p} \leq 0,05)$ con U. europaeus que con el testigo (Figura 1A), sin presentar diferencias significativas con L. albus. Estas diferencias se pueden explicar por el aporte de $\mathrm{N}$ y K al suelo generado por U. europaeus (Tabla 2). Dzida et al. (2012) señalan que el $\mathrm{N}$ es el principal responsable del crecimiento de $B$. vulgaris var. Cicla. Al respecto, Dzida (2004) y Kołota y Czerniak (2010) señalan que a mayor contenido de $\mathrm{N}$ mayor es el rendimiento en materia verde.

Por otra parte, cuando el vegetal absorbe exceso de nitratos, el valor nutritivo de la planta es menor (Dzida et al., 2012). Según Miceli y Miceli (2014) dosis de $\mathrm{N}$ superiores a $200 \mathrm{~kg} \mathrm{ha}^{-1}$ se traducen en acumulación de nitratos en $B$. vulgaris $\mathrm{L}$. var. Cicla, afectando su calidad nutricional.

En la Figura 1B se observa el efecto de los abonos verdes sobre el contenido de proteína, el que fue $14 \%$ superior $(\mathrm{p} \leq 0,05)$ al testigo. Ello puede estar relacionado con el aporte de nutrientes al suelo (Tabla 2), especialmente de $\mathrm{N}$ y K generado en el proceso de descomposición de los abonos verdes. $\mathrm{Al}$ respecto, Escalona et al. (2009) señalan que con la aplicación de una fuente nitrogenada al suelo se puede obtener mayor contenido de nitrato en MV y MS en hojas de lechuga sin sobrepasar las concentraciones límites por lo cual podrían utilizarse sin que ocasionen riesgo a la salud humana.

La inexistencia de diferencias en MV y PC entre L. albus y U. europaeus avala el uso de U. europaeus

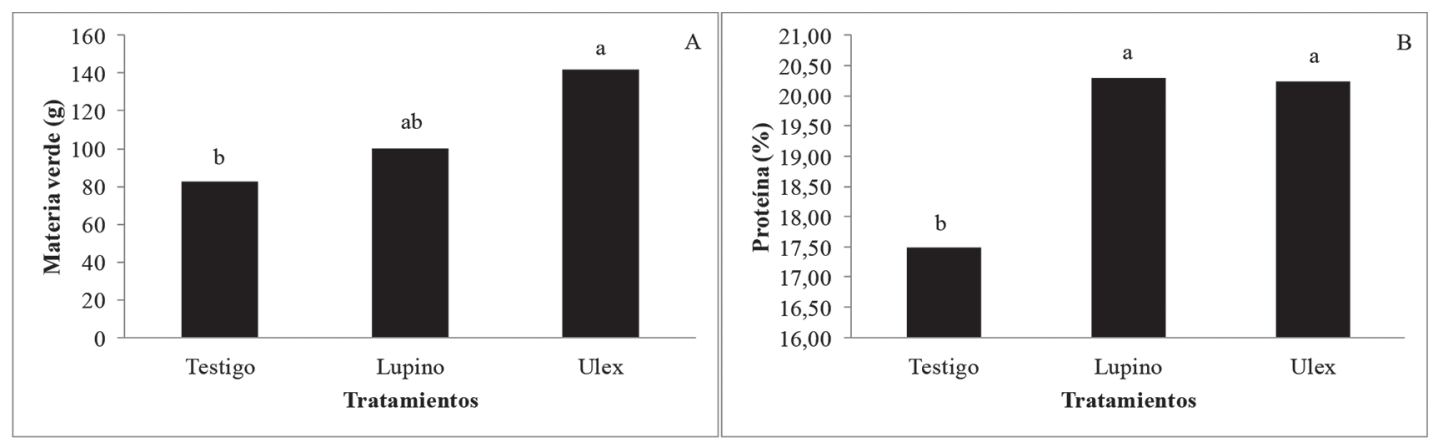

Figura 1. Producción de materia verde (A) y proteína (B) en acelga tratada con abono verde de U. europaeus y de L. albus evaluados junto a un testigo sin incorporación de material vegetal. Letras distintas en cada barra indican diferencias estadísticamente significativas de acuerdo con la prueba de Tukey $(\mathrm{p} \leq 0,05)$. 
Tabla 2. Análisis químico de suelo de cada tratamiento.

\begin{tabular}{lccc}
\hline Parámetro & Testigo & L. albus & U. europaeus \\
\hline P Olsen $\left(\mathrm{mg} \mathrm{kg}^{-1}\right)$ & 60 & 60 & 62 \\
K disponible $\left(\mathrm{mg} \mathrm{kg}^{-1}\right)$ & 651,3 & 717,6 & 822,9 \\
pH en agua & 6,19 & 6,05 & 5,88 \\
Materia orgánica $(\%)$ & 11,35 & 11,57 & 11,03 \\
Ca intercambiable $\left(\mathrm{cmol}+\mathrm{kg}^{-1}\right)$ & 13,68 & 13,85 & 15,11 \\
Mg intercambiable $\left(\mathrm{cmol}+\mathrm{kg}^{-1}\right)$ & 3,41 & 3,42 & 3,44 \\
Na intercambiable $\left(\mathrm{cmol}+\mathrm{kg}^{-1}\right)$ & 0,34 & 0,33 & 0,36 \\
K intercambiable $\left(\mathrm{cmol}+\mathrm{kg}^{-1}\right)$ & 1,67 & 1,84 & 2,11 \\
Al intercambiable $\left(\mathrm{cmol}+\mathrm{kg}^{-1}\right)$ & 0,016 & 0,017 & 0,017 \\
Saturación de Al $(\%)$ & 0,08 & 0,09 & 0,08 \\
CICE $\left(\mathrm{cmol}+\mathrm{kg}^{-1}\right)$ & 19,12 & 19,46 & 21,04 \\
Suma de bases $\left(\mathrm{cmol}+\mathrm{kg}^{-1}\right)$ & 19,1 & 19,44 & 21,02 \\
N $\left(\mathrm{cmol}+\mathrm{kg}^{-1}\right)$ & 80 & 87 & 94 \\
\hline
\end{tabular}

Fuente: análisis de Laboratorio y Servicios Agropecuarios (Labosel) Universidad Católica de Temuco. Mayo del 2012.

como abono verde. Además, si se considera la gran cantidad de biomasa de $U$. europaeus disponible, se constituye en una oportunidad que no se ha abordado.

En la Tabla 3 se puede apreciar que no existen diferencias significativas de EE, FC y cenizas evaluados entre los tratamientos, así como tampoco en la producción de biomasa. Sin embargo, existe mayor producción de MS, EE y de cenizas con abono verde de $U$. europaeus.

En la Tabla 2 se resumen los resultados del análisis de suelo. Es necesario destacar que el ensayo se realizó sobre un suelo fértil, con alto nivel de N, P, K y suma de bases, adecuado $\mathrm{pH}$ y baja saturación de aluminio. Debido a esta condición, los valores $\mathrm{EE}, \mathrm{FC}$, cenizas y producción de biomasa encontrados son altos, incluso en el testigo. No obstante, el abono verde de U. europaeus generó una condición de suelo más favorable que los otros tratamientos. Los parámetros que dan cuenta de ello son el incremento de $\mathrm{N}$ en $14 \mathrm{mg} \mathrm{kg}^{-1}$, $\mathrm{P}$ en $2 \mathrm{mg} \mathrm{kg}^{-1}$, $\mathrm{K}$ en $171 \mathrm{mg} \mathrm{kg}^{-1}$, suma de bases y K intercambiable respecto del testigo.
La incorporación y posterior degradación de abonos verdes pueden aumentar el $\mathrm{P}$ disponible para los cultivos posteriores (Cavigelli y Thien, 2003). Esto es debido a que los abonos provocan un incremento de la cantidad y actividad de microorganismos en el suelo (Navarro y Navarro, 2003).

El $\mathrm{K}$ corresponde al macronutriente que se incrementó en mayor nivel, tras la incorporación de U. europaeus. Navarro y Navarro (2003) señalan que este elemento pasa al suelo por la descomposición de restos vegetales. En U. europaeus la concentración de K llega al 3,1\% foliar (Howe et al., 1988), lo que explica los resultados encontrados.

El contenido de materia orgánica no se vio afectado, probablemente debido a que el material incorporado fue poco lignificado y con alto contenido de N. Esto es especialmente importante en el caso de las leguminosas, ya que su incorporación como abono verde puede contribuir con grandes cantidades de $\mathrm{N}$ en el sistema (Zotarelli et al., 2012).

El menor $\mathrm{pH}$ en el suelo tratado con abonos verdes puede tener su origen en las reacciones

Tabla 3. Producción de biomasa y parámetros nutricionales medidos en acelga.

\begin{tabular}{lccccc}
\hline Tratamiento & MS g & $\%$ MS & $\%$ EE & $\%$ FC & $\%$ Cenizas \\
\hline Testigo & $10,78 \pm 1,27$ a & $12,61 \pm 0,22 \mathrm{a}$ & $1,40 \pm 0,12$ a & $9,15 \pm 0,21$ a & $13,16 \pm 0,54 \mathrm{a}$ \\
L. albus & $12,16 \pm 0,88 \mathrm{a}$ & $12,21 \pm 0,84 \mathrm{a}$ & $1,52 \pm 0,07$ a & $9,00 \pm 0,07$ a & $14,45 \pm 0,11 \mathrm{a}$ \\
U. europaeus & $15,11 \pm 2,72 \mathrm{a}$ & $10,47 \pm 0,94 \mathrm{a}$ & $1,65 \pm 0,13 \mathrm{a}$ & $9,11 \pm 0,15 \mathrm{a}$ & $14,69 \pm 0,49 \mathrm{a}$ \\
\hline
\end{tabular}

MS: materia seca; EE: extracto etéreo; FC: fibra cruda.

Los datos representan el promedio \pm el error estándar. Letras distintas en cada columna indican diferencias estadísticamente significativas para la prueba de Tukey $(\mathrm{p} \leq 0,05)$. 
de intercambio de iones y de hidrólisis de los componentes inorgánicos y orgánicos, debido a que la materia orgánica producto de la descomposición de abonos verdes es una fuente importante de aniones esenciales para las plantas como $\mathrm{NO}_{3}^{-}$, $\mathrm{PO}_{4}^{-3}, \mathrm{BO}_{4}^{-2}, \mathrm{MoO}_{4}^{-3}$ y $\mathrm{Cl}^{-}$(Mosavi et al., 2009).

\section{Conclusiones}

La incorporación de $U$. europaeus aumenta la producción de materia verde y proteína cruda en acelga, no así la producción de materia seca. Por lo tanto, es posible utilizar biomasa de $U$. europaeus como material vegetal para incorporar al suelo en la forma de abono verde, con resultados similares a los encontrados en L. albus.

El ensayo se realizó sobre un suelo fértil y se encontraron resultados favorables, lo que permite inferir que este abono tendrá un mayor impacto en suelos de menor fertilidad; basado en un mayor efecto marginal en las variables evaluadas, apreciándose además, un incremento de los macronutrientes primarios del suelo.

\section{Literatura Citada}

AOAC.

2011. Official methods of analysis of AOAC international. $18^{\text {th }}$ ed. Wisconsin, Madison: AOAC International.

Cavigelli, M.; Thien, S.

2003. Phosphorus bioavailability following incorporation of green manure crops. Soil Science Society of America Journal, 67: 1186-1194.

Chi-Peng, C.; Chih-Hsin, C.; Yu-Hsuan, H.; Chien-Ten, C.; Chao-Ming, L.; Oleg, M.; Liang-Jen, F.; Yaw-Win, Y.

2014. Converting leguminous green manure into biochar: changes in chemical composition and $\mathrm{C}$ and $\mathrm{N}$ mineralization. Geoderma, 232-234: 581-588.

Crews, T.E.; Peoples, M.B.

2004. Legume versus fertilizer sources of nitrogen ecological tradeoffs and human need. Agriculture, Ecosystems \& Environment, 102: 279-297.

Dzida, K.

2004. Effect of nitrogen potassium fertilization on yields of leaf beet (Beta vulgaris L. var. Cicla L.) and concentration of nutrients in the growth medium. Roczniki Akamemii Rolniczej w Poznaniu, 37: 55-60.

Dzida, K.; Zbigniew, J.; Michalojc, Z.

2012. Effect of nitrogen fertilization on the yield and nutritive value of Beta vulgaris L. Journal of Elementology, 17: 19-29.

Eriksen, J.

2005. Gross sulphur mineralization-immobilization turnover in soil amended with plant residues. Soil Biology \& Biochemistry, 37: 2216-2224.

Escalona, A.; Santana, M.; Acevedo, I.; Rodríguez, V.; Merú, L. 2009. Efecto de las fuentes nitrogenadas sobre el contenido de nitratos y lecturas "spad" en el cultivo de lechuga. Agronomía Tropical, 59(1): 99-105.

García, M.; Treto, E.; Álvarez, M.

2002. Época de siembra más adecuada para especies promisorias de abonos verdes en las condiciones de Cuba. Cultivos Tropicales, 23(1): 5-14.

Hill, R.L.; Ireson, J.; Sheppard, A.W.; Gourlay, A.H.; Norambuena, H.; Markin, G.P.; Kwong, R.; Coombs, E.M.

2008. A global view of the future for biological control of gorse, Ulex europaeus L. In: Proceedings of the $12^{\text {th }}$ International Symposium on Biological Control of Weeds. 22-27 April
2007, La Grande Motte, France. CABI, Wallingford, UK. pp. 680-686.

Howe, J.C.; Barry, T.; Popay, A.

1988. Voluntary intake and digestion of gorse (Ulex europaeus) by goats and sheep. The Journals of Agricultural Science, 111: 107-114.

Jensen, C.R.; Joernsgaard, B.; Andersen, M.N.; Christiansen, J.L.; Mogensen, V.O.; Friis, P.; Petersen, C.T.

2004. The effect of lupins as compared with peas and oats on the yield of the subsequent winter barley crop. European Journal of Agronomy, 20: 405-418.

Kołota, E.; Czerniak, K.

2010. The effects of nitrogen fertilization on yield and nutritional value of swiss chard. Acta Scientiarum Polonorum. Hortorum Cultus, 9(2): 31-37.

López, N.; López, A.

2012. Uso de un sustrato alternativo a la turba para la producción viveristica de plantas horticolas y aromaticas. Recursos Rurais, 8: 31-37.

Maccarter, L.E.; Gaynor, D.L.

1980. Gorse: A subject for biological control in New Zealand. New Zealand Journal of Experimental Agriculture, 8(3-4): 321-330.

Meeklah, A.

1979. Controlling gorse. New Zealand Journal Agriculture, 139: 51-53.

Mosavi, S.B.; Jafarzadeh, A.A.; Nishabouri, M.R.; Ostan, S.H.; Feiziasl, V.

2009. Application of rye green manure in wheat rotation system alters soil water content and chemical characteristics under dryland condition in Maragheh. Pakistan Journal of Biological Sciences, 12: 178-182.

Miceli, A.; Miceli, C.

2014. Effect of nitrogen fertilization on the quality of swiss chard at harvest and during storage as minimally processed produce. Journal of Food Quality, 37(2): 125-134.

Navarro, S.; Navarro, G.

2003. Química agrícola, el suelo y los elementos químicos esenciales para la vida vegetal. $2^{\text {da }}$ ed. Madrid, España: Mundi-Prensa libros S.A. 487 p. 
Norambuena, H.; Martinez, G.; Carillo, R.; Neira, M. 2007. Host specificity and establishment of Tetranychus lintearius (Acari: Tetranychidae) for biological control of gorse (Ulex europaeus). Biological Control, 26: 40-47.

Quiroz, C.; Pauchard, A.; Marticorena, A.; Cavieres, L. 2009. Manual de plantas invasoras del Centro-Sur de Chile. Concepción, Chile: Laboratorio de Invasiones Biológicas (LIB), Instituto de Ecología y Biodiversidad (IEB), Facultad de Ciencias Forestales, Universidad de Concepción. 45 p. Rosolem, C.A.; Foloni, J.S.S.; Tiritan, C.S.

2002. Root growth and nutrient accumulation in cover crops as affected by soil compaction. Soil \& Tillage Research, 65: 109-115.
SAS.

2003. JMP User's Guide, Release 5.0.1. Cary, NC: SAS Institute.

Tejada, M.; Gonzalez, J.L.; García-Martínez, A.M.; Parrado, J. 2008. Application of a green manure and green manure composted with beet vinasse on soil restoration: effects on soil properties. Bioresource Technology, 99 (11): 4949-4957.

Zotarelli, L.; Zatorre, N.P.; Boddey, R.M.; Urquiaga, S.; Jantalia, C.P.; Franchini, J.C.; Alves, B.J.R.

2012. Influence of no-tillage and frequency of a green manure legume in crop rotations for balancing $\mathrm{N}$ outputs and preserving soil organic C stocks. Field Crops Research, 132: 185-195. 